\section{The Cambridge Handbook of The Learning Sciences}

Edited by R. Keith Sawyer. Cambridge University Press 2006.648pp. $£ 27.00$ (pb). ISBN 0521607779

We live in a knowledge-creating culture, where most of us regularly flip-flop between being learner and teacher. But we inhabit a bit of a vacuum when it comes to knowledge about knowledge. Few of us read medical education journals and the new generation of educational givens has something of the liberation movement about it, reacting against the conformity of lectures rather than emerging from an evidence base. Knowing how we get to know has suddenly become important though, since, in common with North America and The Netherlands, we are about to embark on a massive reform of postgraduate medical education partly marketed as being based on contemporary learning theory.

So, Keith Sawyer's multi-author book on the relatively new discipline of 'learning science' is timely. Most of us would certainly benefit from being more literate about what goes on in the learning environment and how this contributes to improved learner performance. A limitation of Sawyer's book is that it is aimed mainly at school educationalists, with no reference to learning in medical environments. On the other hand it is a rich source of ideas and evidence on effective learning, with many lessons for psychiatry.

For example, there is a review of the nature of expertise. If you aspire to turn novices into experts, you have to have a pretty good idea of what an expert is. Also how they differ from novices, how you describe that difference in ways that can be translated into measurable outcomes and how you construct a learning environment in which the necessary skills can be acquired. Differences that emerge across a range of disciplines are the expert's ability in 'noticing' and in pattern recognition; skills which affect the ability to rapidly identify problems and opportunities to act on them. The knowledge of experts also tends to be connected and organised around important ideas for their discipline. The lessons for learning include helping novices reflect on their own thinking, enabling them to gain deeper conceptual understanding and exposing them to increasingly complex social and technical environments.

A review of the evidence for what works best underlines the importance of learners being active participants. They need to build new knowledge onto existing knowledge, externalise and articulate their unformed and still developing understanding (articulating and learning go hand in hand), and engage in reflection, which is so critical for professionals working in complex practice. There is also a review of the importance of 'arguing to learn', based on the idea that science advances not by the accumulation of facts, but through debate and argumentation. The justification is that arguing involves elaboration, reasoning and reflection, all of which contribute to deep conceptual learning. Exposure to this so called 'collaborative argumentation' helps professionals think critically and independently about important issues and contested values.

An interesting issue, particularly for psychiatrists, is the role of social context on learning. This is one area that medicine has always done well in, mainly because it has retained a largely apprenticeship model, the preferred way of learning expertise throughout recorded history. The so-called 'cognitive apprenticeship' model emphasises a focus on cognitive skills and processes and involves learning conceptual and factual knowledge used in a variety of contexts to solve real-world problems. Importantly, the apprenticeship model remains intact in the new foundation and run-through training programmes, where for the first time it is reinforced by a formal workplace-based assessment programme.

The Cambridge Handbook of The Learning Sciences is a bit like the Economist; a little over-inclusive for most, but a really good review of those parts of the world you are interested in. Curiously, although there are sections on information technology and learning communities on the internet, as well as a fascinating piece on the importance of enabling conceptual change to take place in learners, there is virtually nothing on assessment. However, there is a tantalising plea to avoid the tendency to assess well-established routines and schemas and instead to examine 'adaptive expertise', or the ability to learn in a knowledge-rich environment. This connects with the idea that all students and trainees are seen as members of a knowledge-building community, where the aim is idea improvement as an alternative to progress towards a 'truth'. And of course in a post-modern world there is no such thing as truth, only different versions of it. One of which is John Dewey's version written in 1938 and quoted in the Cambridge Handbook: 'Education is not preparation for life; education is life'.

Nick Rose Department of Psychiatry, University of Oxford, Warneford Hospital, Oxford OX3 7JX, UK. Email: nick.rose@oxmhc-tr.nhs.uk doi: 10.I192/bjp.bp.106.029678 